\title{
Early Effects of Unfractionated Heparin on Clinical and Radiological Signs and D-dimer Levels in Patients With COVID-19 Associated Pulmonary Embolism: an Observational Cohort Study
}

\section{Lea Imeen van der Wal ( $\sim$ L.I.van_der_wal@lumc.nl )}

Department of Intensive Care Medicine Leiden University Medical Center Postbus 9600, 2300 RC Leiden, The Netherlands

\section{Research}

Keywords: COVID-19, Pulmonary Embolism, Unfractionated Heparin, D-dimer

Posted Date: November 2nd, 2020

DOl: https://doi.org/10.21203/rs.3.rs-99738/v1

License: (c) (1) This work is licensed under a Creative Commons Attribution 4.0 International License. Read Full License 


\section{Abstract}

Background: Pulmonary embolism is a frequent complication in patients with Coronavirus disease 2019 (COVID-19). The pathogenesis of COVID-associated activation of coagulation is not fully understood and appears to be different from disseminated intravascular coagulation (DIC) in patients with sepsis. As the pathophysiology of coagulation in COVID-patients is unknown, it is uncertain whether unfractionated heparin (UFH), or anticoagulation in general, is effective in the attenuation of the procoagulant state. The aim of this study is to determine the effects of intravenous unfractionated heparin on clinical, radiological and laboratory parameters in patients with COVID-19 and acute pulmonary embolism (PE).

Methods: We conducted an observational cohort study in 19 Intensive Care Unit (ICU) patients with COVID-19 and computed tomography (CT) scanning proven pulmonary embolism. According to the local protocol, repeated CT-scanning was indicated if no pulmonary improvement was present after a minimum of 7 days following start of anticoagulant treatment. We defined three endpoints: Laboratory markers (d-dimer at day 0 vs day 2), clinical success (resolution of PE at follow up CT scan or discharged alive from ICU) and radiological response (Qanadli index at follow up CT scan vs CT scan at diagnosis $\mathrm{PE})$. Statistical tests used for analysis were a T-test and Wilcoxon Signed Rank test.

Results: Unfractionated heparin resulted in clinical success in at least 14 out of 19 patients. Pulmonary emboli were completely resolved on the follow up computed tomography scans in 5 out of 6 patients and partly resolved in the $6^{\text {th }}$ patient. D-dimer levels decreased on average from $7074 \mathrm{ng} / \mathrm{mL}$ to $4347 \mathrm{ng} / \mathrm{mL}$ $(p=0.001)$ within 48 hours after start of heparin.

Conclusion: In this observational study, we showed a rapid clinical, laboratory and radiological improvement in patients with COVID-19 and proven pulmonary embolism. Standard anticoagulant treatment was effective in this setting, supporting current guideline recommendations.

\section{Background}

The novel severe acute respiratory syndrome coronavirus 2 (SARS-CoV-2) has a firm grip on public health globally since December 2019. To this date, over 35 million people have been infected worldwide with more than 1 million deaths (1). Patients with progressive disease almost invariably show profound pulmonary inflammation and may require mechanical ventilation and prolonged Intensive Care Unit (ICU) admission. Mortality rates in ICU patients can reach up to 50\% (2). Despite adequate thromboprophylaxis the majority of patients are in a prothrombotic state which results in thrombotic complications, mainly pulmonary embolism, in up to $31 \%$ of the cases (3-6).

The pathogenesis of Corona Virus Disease 2019 (COVID-19)-associated activation of coagulation is currently not fully understood. It differs from disseminated intravascular coagulation (DIC) as seen in patients with sepsis. In DIC coagulopathy is initiated by tissue factor leading to consumption of platelets and coagulation factors with thrombocytopenia and prolonged PT and APTT (7). In contrast, in patients with COVID-19, d-dimer levels are high (up to $20.000 \mathrm{ng} / \mathrm{mL}$ or higher), but platelets and coagulation tests 
are normal in most patients suggesting a different mechanism of activation of coagulation and a high rate of fibrin degradation (8). Differences between COVID-19 pulmonary embolism (PE) and non - COVID19 PE have been also been observed in Computed Tomography (CT) findings, suggesting that COVID-19 associated PE has a different phenotype than 'conventional' PE. In COVID-19 patients PE is frequently located in peripheral lung segments and less extensive compared to PE in patients without COVID-19. It has been hypothesized that the coagulopathy in COVID-19 patients may be driven by a local process associated with severe pulmonary inflammation and in situ thrombosis (9).

Established PE is treated with anticoagulants, which often is unfractionated heparin (UFH) in patients in cardiocirculatory shock or respiratory distress (10). As the pathophysiology of coagulation in COVIDpatients is unknown, it is uncertain whether UFH -or anticoagulation in general-is effective in the attenuation of the procoagulant state. Since insufficient treatment of PE can be fatal, this observational study aims to study the effect of UFH on clinical, radiological and laboratory signs of PE in patient with COVID-19.

\section{Methods}

\section{Patient selection}

This observational cohort study was conducted in the ICU of the Leiden University Medical Center (LUMC) in the Netherlands. This study was approved by the Institutional Review Board of the LUMC for COVID-19 studies. Inclusion criteria were age $>18$ years, proven COVID-19 disease by PCR sampling of nasal/oral airway swab, mechanical ventilation, proven PE documented by CT scanning and treatment with unfractionated heparin. Exclusion criteria were: therapeutic doses of UFH within 48 hours prior to the diagnosis of $\mathrm{PE}$, treatment with reperfusion techniques including fibrinolytic drugs or no data on d-dimer levels prior to the start of UFH therapy. Standard treatment included prophylactic low molecular weight heparin (LMWH) with nadroparin $2850 \mathrm{IU} /$ day subcutaneously or $5700 \mathrm{IE} /$ day if bodyweight $>90 \mathrm{~kg}$. Double prophylactic LMWH was defined as nadroparin $5700 \mathrm{IE} /$ day. According to the local protocol, repeated CT-scanning was indicated if no pulmonary improvement was present after a minimum of 7 days following start of anticoagulant treatment.

\section{Clinical and biological data}

Data was collected for a maximum of 21 days or until ICU discharge. The following clinical and laboratory data were extracted from medical records: age, sex, year of birth, body mass index (BMI), date of ICU admission, date of ICU discharge, reason for discharge, condition 28 days after admission, starting time of UFH therapy and available D-dimer levels which were measured as a part of routine care every day. D-dimer has been measured in citrated plasma on a STA-R MAX analyzer with latex-based immunoturbidimetric reagents from STAGO BNL, Leiden, the Netherlands. Successful treatment was defined as either no PE on follow-up CT or survival at ICU discharge.

\section{CT data acquisition and analysis}


A CT-scan was performed in case of suspected PE. Standard contrast-enhanced CT pulmonary angiography (CTPA) was performed using a 320-MDCT scanner (AquilionONE, Canon) with collimation of $80 \times 0.5 \mathrm{~mm}$ section thickness. Rotation time was 0.275 second, with a helical pitch of 65 . Tube current was with automated exposure control and tube voltage was $100 \mathrm{kVp}$. The amount of iodinated contrast (Xenetix 350 ) was $50-80 \mathrm{~mL}$ with a flow of $4.5-6.0 \mathrm{~mL} / \mathrm{s}$ followed by a and saline flush of $45-50 \mathrm{~mL}$. Images were reconstructed with 1 and $3 \mathrm{~mm}$ thickness using AIDR 3D enhanced technique. All scans were evaluated on a dedicated PACS workstation by a radiologist with $>20$ years of experience in chest CT. The diagnosis of PE was established on CTPA based on filling defect in a pulmonary artery. The thrombus load within the pulmonary arteries was determined by using the Qanadli obstruction index and calculated as percentage vascular obstruction (11). Parenchymal lung tissue involvement regarding pathology due to COVID-19 infection and pre-existing pathology, comprising a composition of groundglass- or alveolar consolidation, atelectasis, emphysema, and fibrosis, was visually assessed by evaluation of axial, coronal, and sagittal reconstructions and expressed as percentage.

\section{Outcomes}

We defined three endpoints: Laboratory markers (d-dimer at day 0 vs day 2), clinical success (resolution of PE at follow up CT scan or discharged alive from ICU) and radiological response (Qanadli index at follow up CT scan vs CT scan at diagnosis PE).

Severe bleeding was defined according to the definition of the International Society on Thrombosis and Haemostasis (ISTH) of 'major bleeding in non-surgical patients' (12).

\section{Statistical analysis}

Statistical analysis was performed by using IBM SPSS statistics version 25 . Normality of the data was tested using the Shapiro-Wilk-test. Normally distributed data are presented as means with standard deviation (SD); data outside normal distribution are presented as medians with interquartile range (IQR). Categorical variables are presented as numbers and percentages. To calculate a significant difference between the two groups, a T-test and Wilcoxon Signed Rank test was used. A two-sided $p<0.05$ was considered statistically significant. The graphs are created using GraphPad Prism version 8.

\section{Results}

In total, between March 15 and May $1^{\text {st }} 2020,90$ patients were admitted to the ICU with confirmed COVID19. Nineteen patients fulfilled the in- and exclusion criteria (Figure 1). All patients were mechanically ventilated and had PE proven by CT-scanning. Baseline, radiological and laboratory characteristics are shown in Table 1 and 2. All patients received either prophylactic, or double prophylactic doses of thromboprophylaxis with nadroparin (low molecular weight heparin (LMWH)) which was switched to therapeutic unfractionated heparin when the diagnosis of PE was confirmed.

\section{Radiological outcome}


The mean Qanadli index of the CT-scans before start of heparin was 17.5\% (SD 10.8\%), with the segmental artery being the most frequent location of the thrombi. Follow-up CT-scans were performed in 6 patients with an average follow-up of 18 days. The Qanadli index decreased significantly from baseline to follow-up ( $p=0.03$ for difference with baseline CT-scan). A Qanadli index decrease to $0 \%$ was observed in 5 patients and a decrease to $5 \%$ was seen in the remaining patient. Two patients had a third follow-up CT-scan. In one patient there was already no remainder of PE on the first follow-up CT-scan and in the other patient the CT-scan remained stable with a Qanadli index of $5 \%$ at 25 days. Both were still receiving heparin at the time of the second follow-up CT-scan.

\section{Clinical outcome}

In at least 14 (74\%) patients, UFH treatment was successful: in 6 patients (32\%) PE was found to be completely resolved on follow-up CT, whereas 8 patients (42\%) were discharged from the ICU following clinical improvement. One patient died in the ICU without follow-up CT-scan to evaluate treatment. From the remaining $4(21 \%)$ patients it is unknown whether treatment was successful, because they were transferred to another ICU.

At 28 days after admission $9(47,4 \%)$ patients were still in the ICU (including 3 of the 4 patients that were eventually transferred to another ICU), 7 (36.8\%) patients were discharged from the ICU to either the nursing ward $(n=5,26.3 \%)$, a rehabilitation center $(n=1,5.3 \%)$ or home $(n=1,5.3 \%)$. In total, $2(10.5 \%)$ patients died (of which 1 had clinical improvement on the follow-up CT) in the ICU and 1 (5.3\%) was transferred to another ICU.

In our cohort, 6 patients (32\%) suffered from bleeding complications, of which 2 patients $(10.5 \%)$ were classified as severe bleeding. These severe bleedings were located in the lung $(n=1)$ and the lower gastrointestinal tract $(n=1)$. None of these bleedings resulted in death.

\section{Laboratory outcome}

D-dimer levels from all patients from approximately 2 days before the start of heparin until 21 days after start heparin or until ICU-discharge are shown in Figure 2.

The percentage change of D-dimer levels in relation to start of heparin per 24-hours period from two days before UFH to 2 days after start of UFH are shown in Figure 3. All blood samples used to determine Ddimer levels were taken at $6 \mathrm{AM}$. Therefore $\mathrm{T}=0$ in this graph represents the $\mathrm{D}$-dimer taken at $6 \mathrm{AM}$ at the day UFH was started. The actual start of UFH varied for every patient. Mean start of heparin was 9 hours (SD 4.9) after blood sampling. From the first time point, from day -2 to day -1 , the D-dimer dropped on average 6.7\% (SD 26.9) after which, from day -1 to day 0 , it increased $5.6 \%$ (SD 25.6). The first day after start of UFH, a mean drop of $17.9 \%$ (SD 19.4) and the second day a drop of $14.6 \%$ (SD 15.9) was seen. The average D-dimer at day 0 was $7074 \mathrm{ng} / \mathrm{mL}$ compared to $4347 \mathrm{ng} / \mathrm{mL}$ at day $2(\mathrm{p}=0.001)$. The mean difference from day 0 to day 2 was $-2810 \mathrm{ng} / \mathrm{mL}(95 \% \mathrm{Cl}-721 \mathrm{ng} / \mathrm{mL}$ to $-4347 \mathrm{ng} / \mathrm{mL})$. 


\section{Discussion}

In this study we show that clinical and radiological signs of PE and plasma D-dimer levels decreased after administration of UFH in patients with COVID-19 and PE. This is the first study on the effect of heparin (either UFH or LMWH) on thrombosis in COVID-19 patients. Earlier, Tang and others (13) studied the effect of LMWH in 449 COVID-19 patients. They found an association between increasing D-dimer and higher mortality in non-LMWH treated patients. Also, a reduced mortality was seen in patients with coagulopathy who were treated with LMWH compared with patients with coagulopathy who were not treated with LMWH ( $40 \%$ vs $64.2 \%, p=0.029$ ). However, the effect of UFH on D-dimer levels or PE resolution was not reported.

Despite the fact that our results suggest that therapeutic UFH is an effective treatment of COVID-19 associated PE, thrombo-embolic complications are common despite prophylactic LMWH $(3,14)$. An explanation for this might be the route of administration or the dose. Giving subcutaneous LMWH prophylaxis in the ICU might lead to lower anti-Xa activity by the concurrent use of vasoconstrictors such as norepinephrine (15). Norepinephrine was also administered in most COVID-19 patients in the ICU (data not shown). Furthermore, prophylactic doses of LMWH are lower than therapeutic doses and result in lower anti-Xa activity. Consequently, anticoagulant effects will be lower and may be insufficient to prevent $P E$. It is currently unknown if increasing the doses of $L M W H$ would be beneficial in preventing thrombotic complications in COVID-19 patients. Some authors suggest to treat severe COVID-19 pneumonia, even without serious obstructive signs of pulmonary embolism, with thrombolysis in order to improve oxygenation (16). However, our results show that those measures are not necessary: regular treatment is effective and can resolve PE on short notice. Therefore we support current guideline recommendations to reserve thrombolysis to patients with high-risk PE and apply standard dose thromboprophylaxis.

There have been concerns about a high incidence of Chronic Thrombotic Pulmonary Hypertension (CTEPH) after COVID-19 associated PE, in particular because inflammatory states have been associated with poor thrombus resolution. $(17,18)$. Although our sample size is small, the rapid clot resolution observed in our study suggests that the incidence of CTEPH in COVID-19 associated PE survivors may not be notably increased. Even so, physicians should remain vigilant on the presence of CTEPH in patients treated for COVID-19 associated PE who have not been recovered after a 3-month follow-up period.

In our study population with UFH, 6 out of 19 patients experienced bleeding complications with 2 severe bleeding episodes. To properly outweigh the risk of bleeding and the risk of thrombosis, properly designed randomized controlled trials (RCTs) are needed to establish the optimal dose and route of administration for COVID-19 patients. Currently, several RCTs are underway (19).

Our study had several limitations. The change in D-dimer levels may have been influenced by other factors than administration of UFH. A fall in D-dimer may for instance also reflect an improvement of the inflammatory state. In this uncontrolled observational study we cannot exclude confounding by factors modifying the severity of illness. Therefore, the decline in D-dimers that we found in the two days after 
start of heparin could also be caused by clinical improvement in general. Another limitation is the limited sample size of 19 patients with only 6 patients having had a follow-up CT scan. Strongpoints of the study include the strict protocol in our ICU dictating repeated CT-scanning if no improvement of pulmonary status was present after one week of treatment of UFH, and the meticulous comparison of index and follow-up CTPA scan images.

\section{Conclusion}

In conclusion, we show a considerable clinical and radiological improvement in patients with COVID-19 and proven PE after starting UFH therapy. Standard anticoagulant treatment therefore seems to be effective in this setting, supporting current guideline recommendations.

\section{List Of Abbreviations}

BMI: Body Mass Index

COVID-19: Corona Virus Disease 2019

CT: Computed Tomography

CTEPH: Chronic Thrombotic Pulmonary Hypertension

CTPA: Computed Tomography Pulmonary Angiography

DCTC: Dutch Covid \& Thrombosis Coalition

DIC: Disseminated Intravascular Coagulation

ICU: Intensive Care Unit

IQR: Interquartile Range

ISTH: International Society on Thrombosis and Haemostasis

LMWH: Low Molecular Weight Heparin

LUMC: Leiden University Medical Center

PE: Pulmonary Embolism

RCT: Randomized Controlled Trial

SARS-CoV-2: Severe Acute Respiratory Syndrome Coronavirus 2

SD: Standard Deviation 
UFH: Unfractionated Heparin

\section{Declarations}

\section{Ethics approval and consent to participate}

This study was approved by the Institutional Review Board of the LUMC for COVID-19 studies and performed in accordance with the Declaration of Helsinki. The need for consent was waived by the Institutional Review Board for COVID-19 studies. All data used in this study were collected as part of normal patient care and independent of this research. Also, our database does not contain identifiable information. Asking informed consent in this setting would be disproportionate. Patients and their family are informed in the patient information leaflet given at ICU-admission that they may opt-out from research using patient-data.

Consent for publication

Not applicable

Availability of data and materials

The datasets used and analysed during the current study are available from the corresponding author (L.I.van_der_wal@lumc.nl) on reasonable request.

\section{Competing interests}

MV Huisman received grants from ZONMW, Bayer Health Care, Pfizer-BMS, Boehringer-Ingelheim, Leo Pharma, all outside the submitted work. J Eikenboom received research support from CSL Behring and he has been teacher on educational activities of Roche, all outside the submitted work. FA Klok reports research support from Bayer Health Care, Bristol-Myers Squibb, Boehringer-Ingelheim, MSD, DaiichiSankyo, Actelion, the Dutch thrombosis association, The Netherlands Organisation for Health Research and Development and the Dutch Heart foundation, all outside the submitted work. The authors declare that they have no competing interests.

\section{Funding}

We received funding from ZONMW and the Dutch Thrombosis Association. There was no role of the funding bodies in the design of the study and collection, analysis and interpretation of data and in writing the manuscript.

\section{Authors' contributions}

LIW collected the data and wrote the manuscript. EJ initiated and supervised this study. All authors contributed to the writing of the manuscript and approved the final version. 
Acknowledgements

\section{Collaborating author names from the Dutch COVID \& Thrombosis Coalition (DCTC).}

Lea I. van der Wal ${ }^{1}$, Lucia J.M. Kroft ${ }^{2}$, Lisette F. van Dam ${ }^{3}$, Christa M. Cobbaert ${ }^{4}$, Jeroen Eikenboom³, Menno V. Huisman ${ }^{3}$, Hendrik J.F. Helmerhorst ${ }^{1,5}$, Frederikus A. Klok ${ }^{3}$, Evert De Jonge ${ }^{1}$.

${ }^{1}$ Department of Intensive Care Medicine, Leiden University Medical Center, Leiden, The Netherlands

${ }^{2}$ Department of Radiology, Leiden University Medical Center, Leiden, The Netherlands

${ }^{3}$ Department of Thrombosis and Hemostasis, Leiden University Medical Center, Leiden, The Netherlands

${ }^{4}$ Department of Clinical Chemistry and Laboratory Medicine, Leiden University Medical Center, Leiden, The Netherlands

${ }^{5}$ Department of Anesthesiology, Leiden University Medical Center, Leiden, The Netherlands

Authors' information

Not applicable

\section{References}

1. John Hopkins University \& Medicine: Coronavirus Resource Center [Accessed 6 October 2020]. Available from: https://coronavirus.jhu.edu/.

2. Yang $X, Y u$ Y, Xu J, Shu H, Xia JA, Liu H, et al. Clinical course and outcomes of critically ill patients with SARS-CoV-2 pneumonia in Wuhan, China: a single-centered, retrospective, observational study. The Lancet Respiratory Medicine. 2020;8(5):475-81.

3. Klok FA, Kruip MJHA, Van Der Meer NJM, Arbous MS, Gommers D, Kant KM, et al. Confirmation of the high cumulative incidence of thrombotic complications in critically ill ICU patients with COVID-19: An updated analysis. Thrombosis Research. 2020;191:148-50.

4. Klok FA, Kruip MJHA, Van Der Meer NJM, Arbous MS, Gommers DAMPJ, Kant KM, et al. Incidence of thrombotic complications in critically ill ICU patients with COVID-19. Thrombosis Research. 2020;191:145-7.

5. Lodigiani C, lapichino G, Carenzo L, Cecconi M, Ferrazzi P, Sebastian T, et al. Venous and arterial thromboembolic complications in COVID-19 patients admitted to an academic hospital in Milan, Italy. Thrombosis Research. 2020;191:9-14.

6. Helms J, Tacquard C, Severac F, Leonard-Lorant I, Ohana M, Delabranche X, et al. High risk of thrombosis in patients with severe SARS-CoV-2 infection: a multicenter prospective cohort study. Intensive Care Medicine. 2020;46(6):1089-98. 
7. Levi M, De Jonge $E$, Van Der Poll T. New treatment strategies for disseminated intravascular coagulation based on current understanding of the pathophysiology. Annals of Medicine. 2004;36(1):41-9.

8. Connors JM, Levy JH. COVID-19 and its implications for thrombosis and anticoagulation. Blood. 2020;135(23):2033-40.

9. Van Dam LF, Kroft LJM, Van Der Wal LI, Cannegieter SC, Eikenboom J, De Jonge E, et al. Clinical and computed tomography characteristics of COVID-19 associated acute pulmonary embolism: A different phenotype of thrombotic disease? Thrombosis Research. 2020;193:86-9.

10. Konstantinides SV, Meyer G, Becattini C, Bueno H, Geersing G-J, Harjola V-P, et al. 2019 ESC Guidelines for the diagnosis and management of acute pulmonary embolism developed in collaboration with the European Respiratory Society (ERS). European Heart Journal. 2020;41(4):543603.

11. Qanadli SD, El Hajjam M, Vieillard-Baron A, Joseph T, Mesurolle B, Oliva VL, et al. New CT index to quantify arterial obstruction in pulmonary embolism: comparison with angiographic index and echocardiography. AJR Am J Roentgenol. 2001;176(6):1415-20.

12. Schulman S, Kearon C. Definition of major bleeding in clinical investigations of antihemostatic medicinal products in non-surgical patients. Journal of Thrombosis and Haemostasis. 2005;3(4):692-4.

13. Tang N, Bai H, Chen X, Gong J, Li D, Sun Z. Anticoagulant treatment is associated with decreased mortality in severe coronavirus disease 2019 patients with coagulopathy. Journal of Thrombosis and Haemostasis. 2020;18(5):1094-9.

14. Stoneham SM, Milne KM, Nuttall E, Frew GH, Sturrock BR, Sivaloganathan $\mathrm{H}$, et al. Thrombotic risk in COVID-19: a case series and case-control study. Clinical Medicine. 2020;20(4):e76-e81.

15. Dorffler-Melly J, de Jonge E, Pont AC, Meijers J, Vroom MB, Buller HR, et al. Bioavailability of subcutaneous low-molecular-weight heparin to patients on vasopressors. Lancet. 2002;359(9309):849-50.

16. Wang J, Hajizadeh N, Moore EE, Mclntyre RC, Moore PK, Veress LA, et al. Tissue plasminogen activator (tPA) treatment for COVID-19 associated acute respiratory distress syndrome (ARDS): A case series. Journal of Thrombosis and Haemostasis. 2020;18(7):1752-5.

17. Huisman MV, Barco S, Cannegieter SC, Le Gal G, Konstantinides SV, Reitsma PH, et al. Pulmonary embolism. Nature reviews Disease primers. 2018;4:18028.

18. Ende-Verhaar YM, Cannegieter SC, Vonk Noordegraaf A, Delcroix M, Pruszczyk P, Mairuhu AT, et al. Incidence of chronic thromboembolic pulmonary hypertension after acute pulmonary embolism: a contemporary view of the published literature. The European respiratory journal. 2017;49(2).

19. Tritschler T, Mathieu ME, Skeith L, Rodger M, Middeldorp S, Brighton T, et al. Anticoagulant interventions in hospitalized patients with COVID-19: A scoping review of randomized controlled trials and call for international collaboration. Journal of Thrombosis and Haemostasis. 2020. 


\section{Tables}

Table 1. Baseline characteristics

\begin{tabular}{|c|c|c|}
\hline \multicolumn{3}{|c|}{ Patient characteristics } \\
\hline \multicolumn{2}{|c|}{ Mean Age, year (SD) } & $63(6.6)$ \\
\hline \multicolumn{2}{|c|}{ Male, $\mathrm{n}(\%)$} & $16(84)$ \\
\hline \multicolumn{2}{|c|}{ Mean body mass index, $\mathrm{kg} / \mathrm{m}^{2}$ (SD) } & $27.5(2.8)$ \\
\hline \multicolumn{3}{|c|}{ Thrombosis prophylaxis when admitted at ICU } \\
\hline- & Prophylactic, n (\%) & $16(84.2)$ \\
\hline- & Double prophylactic, n (\%) & $2(10.5)$ \\
\hline- & Therapeutic, n (\%) & $0(0)$ \\
\hline- & Unknown, n (\%) & $1(5.3)$ \\
\hline \multicolumn{3}{|c|}{ Status at 28 days after admission } \\
\hline- & ICU, n (\%) & $9(47.4)$ \\
\hline- & Hospital, n (\%) & $5(26.3)$ \\
\hline- & Death, n (\%) & $2(10.5)$ \\
\hline- & Rehabilitation, n (\%) & $1(5.3)$ \\
\hline- & Home, n (\%) & $1(5.3)$ \\
\hline - & Other, n (\%) & $1(5.3)$ \\
\hline
\end{tabular}

Table 2. Radiological and laboratory characteristics 


\begin{tabular}{|c|c|}
\hline \multicolumn{2}{|l|}{ Radiological presentation } \\
\hline Qanadli index first CT, mean (\%) \pm SD & $17.5( \pm 10.8)$ \\
\hline \multicolumn{2}{|c|}{ Location pulmonary embolism on first index CT } \\
\hline Subsegmental, $\mathrm{n}(\%)$ & $2(10.5)$ \\
\hline Segmental, $\mathrm{n}(\%)$ & $16(84.2)$ \\
\hline Main/lobar, n(\%) & $1(5.3)$ \\
\hline Patients with follow-up CT & 6 \\
\hline \multicolumn{2}{|l|}{ Qanadli index first follow-up CT } \\
\hline $0 \%(n)$ & 5 \\
\hline $5 \%(n)$ & 1 \\
\hline \multicolumn{2}{|l|}{ D-dimers } \\
\hline D-dimers day -2 Median + IQR & $5379 \mathrm{ng} / \mathrm{mL}(2460-10604)$ \\
\hline D-dimers day -1 Median + IQR & 5555 ng/mL (4317-9769) \\
\hline D-dimers day 0 Median + IQR & 6197 ng/mL (4682-9360) \\
\hline D-Dimers day 1 Median + IQR & 4766 ng/mL (3047-7773) \\
\hline D-dimers day 2 Median + IQR & 3665 ng/mL (2470-5437) \\
\hline
\end{tabular}

\section{Figures}




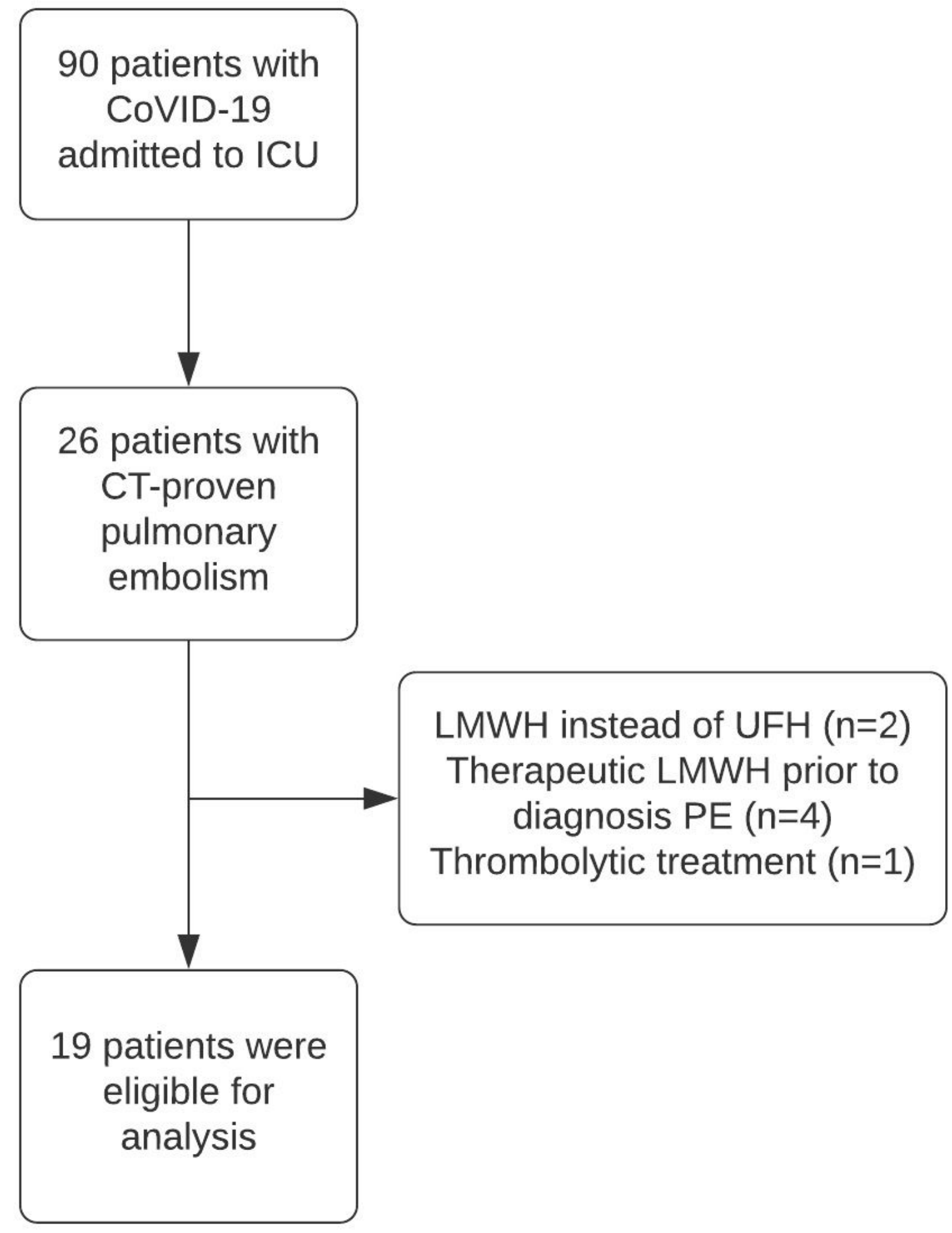

Figure 1

Flow chart of patient selection 


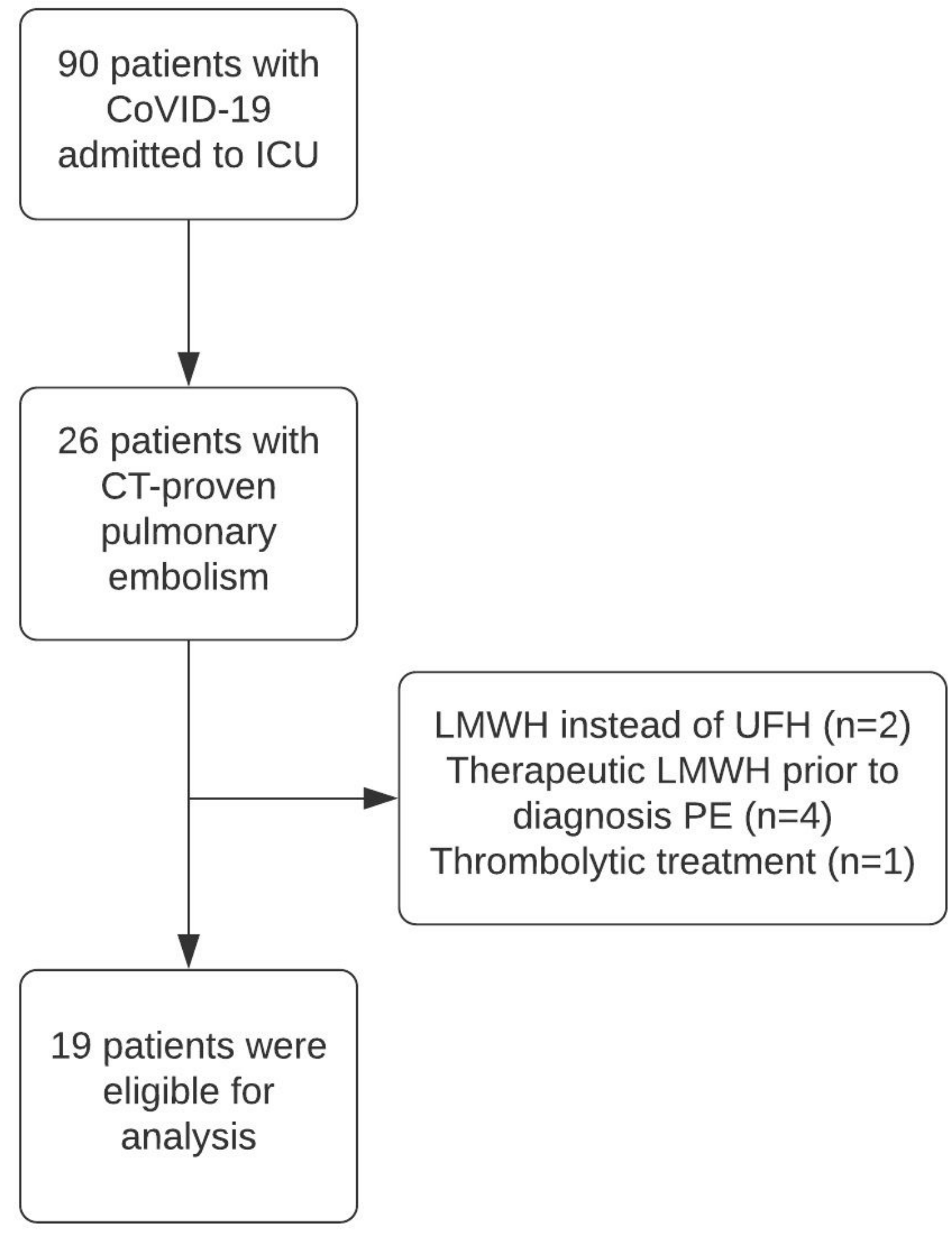

Figure 1

Flow chart of patient selection 


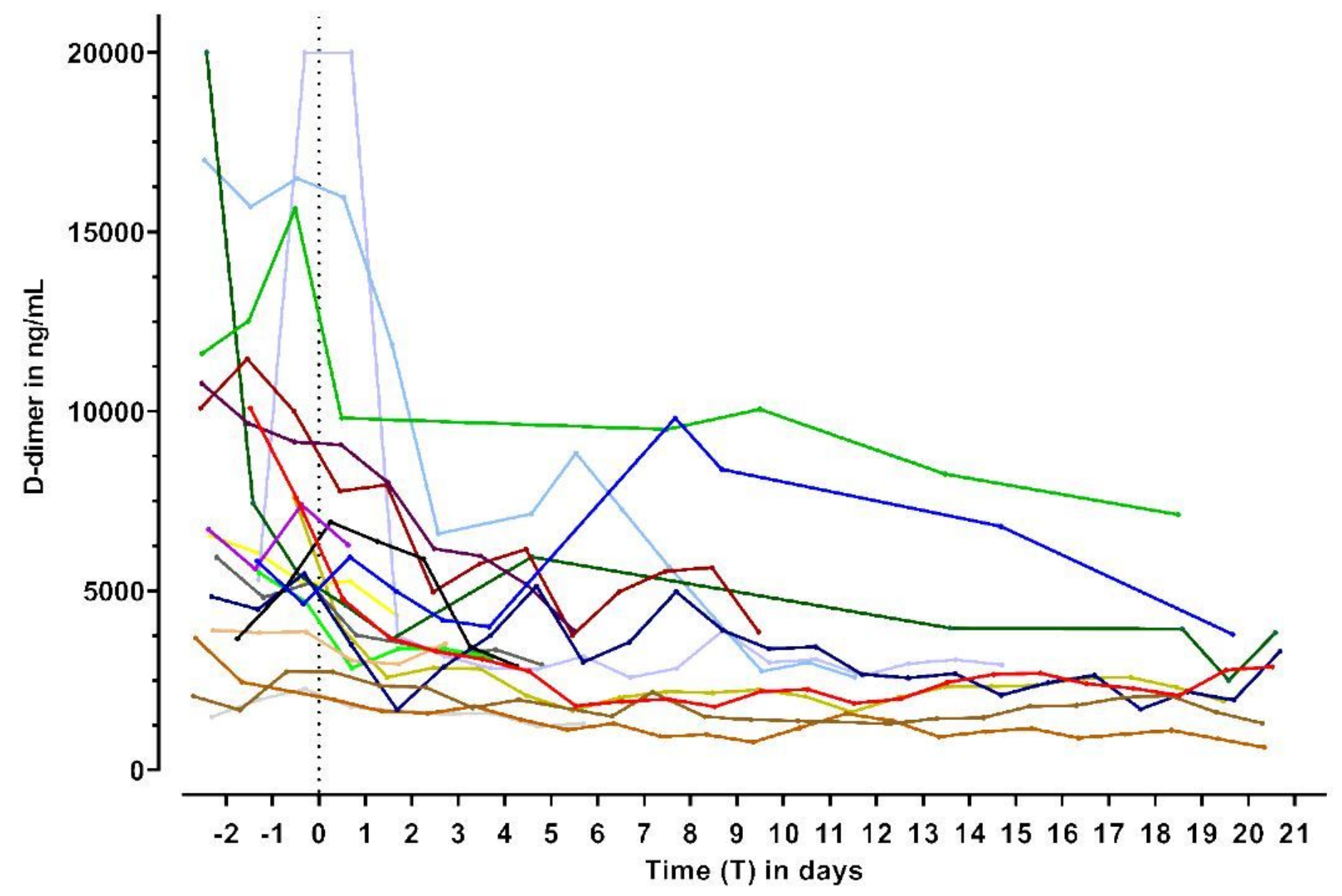

Figure 2

Course of D-dimers before and after start of heparin. D-dimer levels from 2 days before the start of UFH until 21 days after start of UFH or until ICU discharge are shown. T=0 represents the start of heparin for each individual patient, which is marked by the dotted line. 


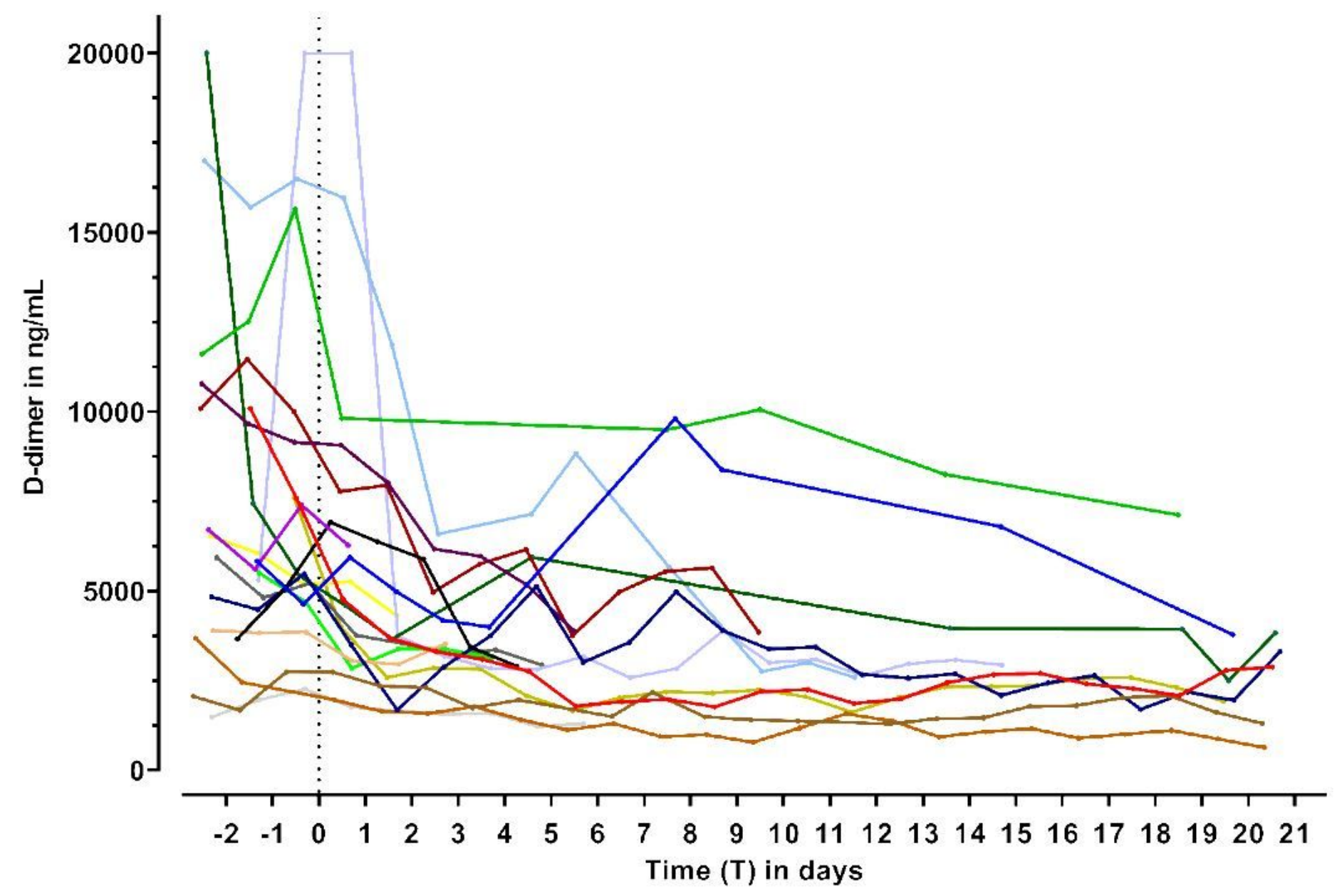

Figure 2

Course of D-dimers before and after start of heparin. D-dimer levels from 2 days before the start of UFH until 21 days after start of UFH or until ICU discharge are shown. T=0 represents the start of heparin for each individual patient, which is marked by the dotted line. 


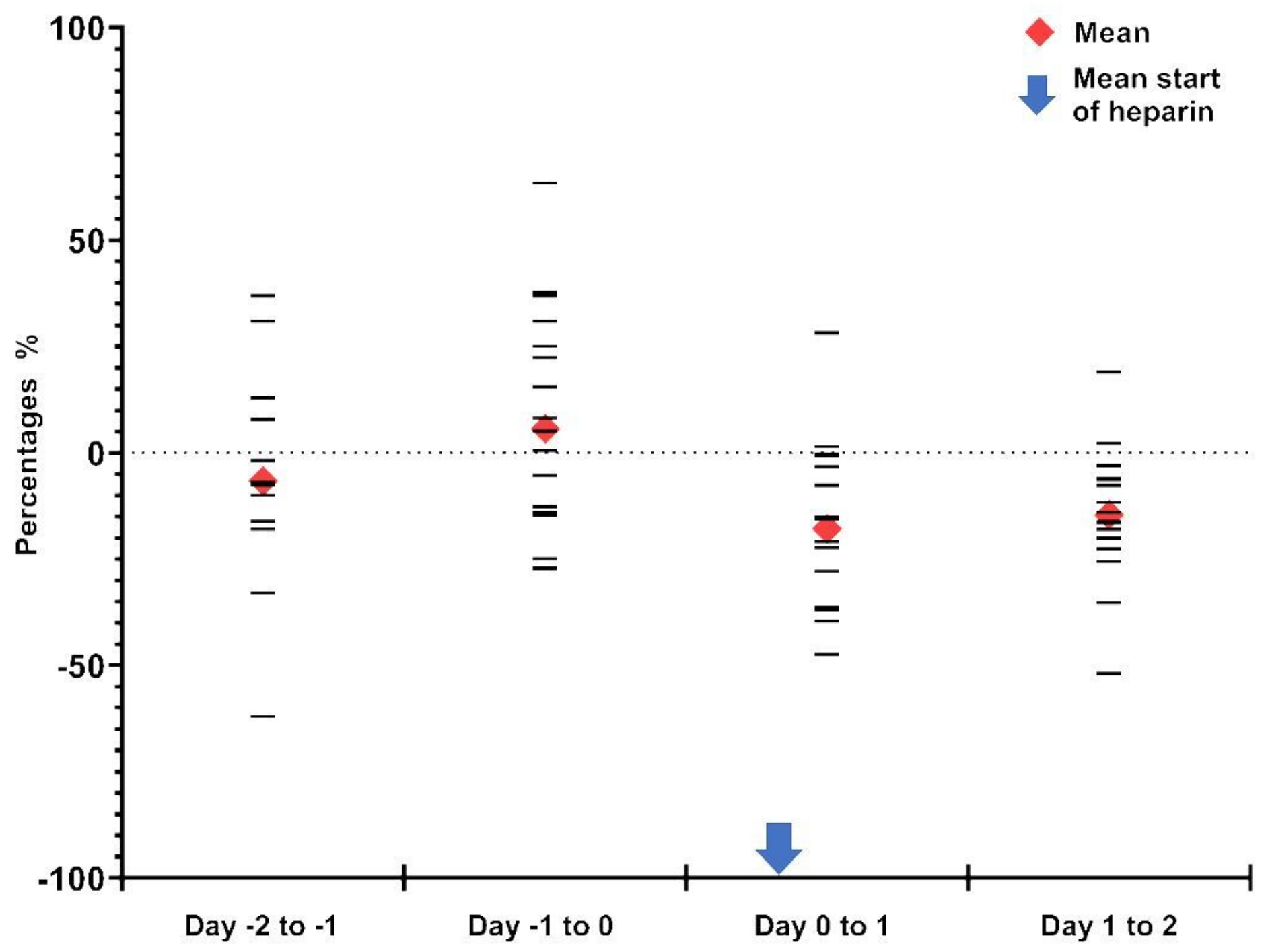

Figure 3

Percentage change of D-dimer levels for different time frames before and after start of heparin. All samples were taken at 6:00 A.M. For every patient $T=0$ represents the time of blood sampling at 6:00 AM of the day that heparin was started. The horizontal lines represent the percentage change in that time frame for each individual patient. The arrow represents the mean actual time of start of heparin. 


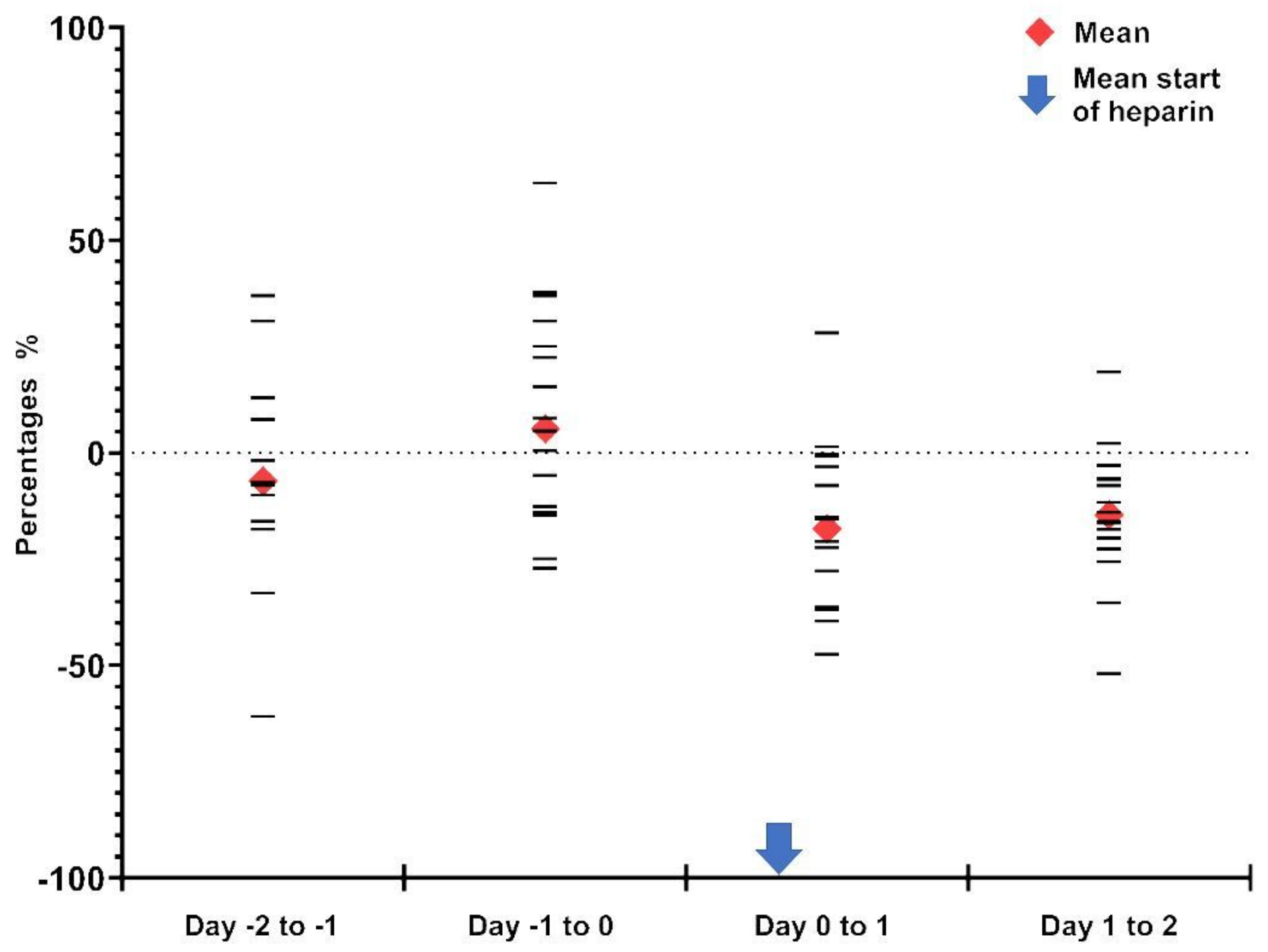

Figure 3

Percentage change of D-dimer levels for different time frames before and after start of heparin. All samples were taken at 6:00 A.M. For every patient $T=0$ represents the time of blood sampling at 6:00 AM of the day that heparin was started. The horizontal lines represent the percentage change in that time frame for each individual patient. The arrow represents the mean actual time of start of heparin. 